\title{
Small Ubiquitin-Related Modifier Is Secreted and Shows Cytokine-Like Activity
}

\author{
Hidetaka Hosono and Hideyoshi YoKosawA* \\ Department of Biochemistry, Graduate School of Pharmaceutical Sciences, Hokkaido University; Sapporo 060-0812, \\ Japan. Received November 7, 2007; accepted February 2, 2008
}

Small ubiquitin-related modifier (SUMO) is a type I ubiquitin-like protein family member and is covalently attached to various target proteins. Through this post-translational modification, SUMO plays important roles in various cellular events. Here, we show that SUMO is secreted from cultured cells in an endoplasmic reticulum (ER)/Golgi-independent manner and that this secretion occurs without covalent binding to target proteins or chain formation. Overexpression experiments using C-terminally truncated mutants of SUMO revealed that the secretion requires the C-terminal sequence. Recombinant SUMO-3 protein was capable of binding to and promoting the proliferation of cultured cells. Thus, we propose that SUMO functions as a cytokine-like molecule extracellularly.

Key words small ubiquitin-related modifier; ubiquitin-like protein; secretion; cytokine

Post-translational modification by small ubiquitin-related modifier (SUMO) has crucial roles in regulation of target proteins. ${ }^{1-3)}$ Four SUMO isoforms (SUMO-1, -2, -3, -4) exist in humans, and SUMO-1, -2 and -3 are covalently linked to lysine residues of target proteins through their unique $\mathrm{C}$ terminal di-glycine motifs. SUMO modification is mediated by a sequential reaction similar to the ubiquitin conjugation system that consists of ubiquitin-activating enzyme (E1), ubiquitin-conjugating enzyme (E2), and ubiquitin ligase (E3). ${ }^{1-3)}$ SUMO is activated by SUMO E1 (Aos-1-Uba2 heterodimer) in an ATP-dependent manner and binds to E1 via a thioester linkage. Next, SUMO is transferred to and binds to SUMO E2 (Ubc9) via a thioester linkage. Finally, SUMO E3s including PIAS family proteins, RanBP2, Topors, and Pc2 promote the transfer of SUMO from E2 to target proteins: SUMO E3, however, is not required for in vitro modification of a certain target protein by SUMO. ${ }^{4,5)}$ Recently, various proteins have been shown to be modified by SUMO: For example, thymine DNA glycosylase is modified by SUMO, which induces a conformation change of the enzyme, resulting in the enhancement of its dissociation from the substrate DNA after base excision. ${ }^{6,7)}$ On the other hand, several proteins, including proliferating cell nuclear antigen (PCNA), inhibitor of nuclear factor- $\kappa \mathrm{B}(\mathrm{I} \kappa \mathrm{B})$, and Huntingtin, are modified by SUMO at the same sites as those for ubiquitin conjugation, resulting in competition between SUMO and ubiquitin for modification, which leads to inhibition of protein degradation by the $26 \mathrm{~S}$ proteasome. ${ }^{8-10)}$

Among ubiquitin and ubiquitin-like proteins that regulate various intracellular events through their attachment to target proteins, ubiquitin-like proteins such as ISG15 (interferon stimulated gene 15) not only function as modifier molecules intracellularly but are also secreted to function as cytokinelike molecules extracellularly. ${ }^{11,12)}$

In this study, we found that SUMO is secreted from cultured cells and that the secreted SUMO molecule shows cytokine-like activity extracellularly.

\section{MATERIALS AND METHODS}

Cell Culture and Transfection HeLa, NIH3T3,
HEK293, H1299, A549, and MCF7 cells were cultured in Dulbecco's modified Eagle's medium (Sigma) supplemented with $10 \%$ calf serum (Hyclone). Transfection in NIH3T3, HEK293, H1299, A549, and MCF7 cells was performed using Metafectene (Biontex) according to the manufacturer's protocol, while that in HeLa cells was performed according to the standard calcium precipitation protocol.

Plasmid Construction The cDNAs of mouse SUMO-1, -3 and -4 were amplified by polymerase chain reaction (PCR). Truncated and point mutants of SUMO-3 were generated by PCR. To generate mammalian expression plasmids of FLAG-tagged SUMO-3 and its mutants, FLAG-tagged green fluorescent protein (GFP), and FLAG-tagged ubiquitin, the respective PCR fragments were subcloned into the pCI-neo3FLAG plasmid that had been generated by inserting three repeats of the FLAG tag sequence into the pCI-neo mammalian expression vector (Sigma). ${ }^{13)}$ The SUMO-1 mammalian expression plasmid, pCGT-T7-SUMO-1, was a gift from Dr. H. Ariga of Hokkaido University and the mammalian expression plasmids of T7-tagged SUMO-3 and -4 were constructed by subcloning of the respective PCR fragments into the pCGT-T7 plasmid.

Preparation of Recombinant Proteins For preparation of recombinant SUMO-3 and its truncated mutants and ubiquitin, the cDNAs of the respective proteins were subcloned into the pGEX6P1 plasmid (GE Healthcare) and transformed into Escherichia coli strain BL21. The expression of glutathione S-transferase (GST)-fusion protein was induced by addition of $0.1 \mathrm{~mm}$ isopropyl 1-thio- $\beta$-D-galactoside for $12 \mathrm{~h}$ at $25^{\circ} \mathrm{C}$ and the cell extract was prepared. The recombinant proteins were isolated from the extract by affinity chromatography on glutathione-Sepharose 4B beads (GE Healthcare) and subsequently by elution through digestion with PreScission protease (GE Healthcare) in buffer containing $20 \mathrm{~mm}$ Tris- $\mathrm{HCl}, \mathrm{pH} 7.0,150 \mathrm{~mm} \mathrm{NaCl}, 0.1 \mathrm{~mm}$ EDTA, and $0.05 \mathrm{~mm}$ dithiothreitol at $4{ }^{\circ} \mathrm{C}$. For preparation of recombinant FLAGtagged SUMO-3 and ubiquitin, the cDNAs of the respective proteins were subcloned into the pGEX-FLAG plasmid that had been generated by inserting three repeats of the FLAGtag sequence into the pGEX6P1 plasmid and transformed into $E$. coli strain BL21. The recombinant proteins were 
prepared as described above.

Preparation of Anti-SUMO-3 Antibody To obtain an antibody against SUMO-3, three tandem repeats of SUMO-3 protein were expressed as a GST-fusion protein and its GST portion was removed as described above. The resulting recombinant SUMO-3 protein was denatured by boiling for $10 \mathrm{~min}$, and a rabbit was immunized three times with $5 \mu \mathrm{g}$ each of the SUMO-3 protein using TiterMax Gold (TiterMax) according to manufacturer's protocol. Anti-SUMO-3 antibody was purified from antiserum by affinity chromatography on a SUMO-3-immobilized resin that had been prepared by immobilizing the SUMO-3 protein to a HiTrap NHS-activated cartridge (GE Healthcare) according to manufacturer's protocol.

SDS-PAGE and Western Blotting HeLa cells that had been transiently transfected with the indicated plasmids and cultured for $36 \mathrm{~h}$ were washed with ice-cold phosphatebuffered saline and lysed with buffer containing $50 \mathrm{~mm}$ Tris- $\mathrm{HCl}, \mathrm{pH} 7.5,150 \mathrm{~mm} \mathrm{NaCl}, 1 \mathrm{~mm}$ EDTA, 0.5\% Triton $\mathrm{X}-100$, and $5 \%$ glycerol. The cell lysate was sonicated for a few seconds and the debris was removed by centrifugation at $15000 \mathrm{rpm}$ for $15 \mathrm{~min}$. The resulting supernatant was subjected to SDS-PAGE. Alternatively, the culture medium (the supernatant in the cell culture) was collected, centrifuged at $5000 \mathrm{rpm}$ for $3 \mathrm{~min}$ to remove any cells, and subjected to SDS-PAGE. The proteins separated by SDS-PAGE were transferred onto a nitrocellulose membrane (Bio-Rad). The membrane was immunoblotted with anti-FLAG M2 (Sigma) and anti-T7 (Novagen) antibodies and subsequently incubated with horseradish peroxidase-conjugated antibodies against mouse immunoglobulin (GE Healthcare), followed by detection with ECL immunoblotting detection reagents (GE Healthcare).

Measurement of Cell Proliferation To evaluate cell proliferation, NIH3T3 cells were cultured in a 96-well plate, up to 5000 cells per well. Recombinant SUMO-3 protein and its truncated mutants and ubiquitin were added to the cells and the cells were cultured for $48 \mathrm{~h}$. The absorbance at $450 \mathrm{~nm}$ due to formation of the formazan dye from tetrazolium salt WST-8, which corresponds to the number of living cells, was measured using a cell counting kit-8 (Dojindo, Japan) according to the manufacturer's protocol.

Binding of SUMO to Cells NIH3T3 cells were incubated on ice for $30 \mathrm{~min}$ in DMEM containing $2 \%$ bovine serum albumin. FLAG-tagged SUMO-3, with or without non-tagged SUMO-3/ubiquitin as a competitor, was added to the cells at a final concentration of $1 \mathrm{ng} / \mathrm{ml}$. The cells were allowed to stand for $10 \mathrm{~min}$ and were then washed with DMEM containing $2 \%$ bovine serum albumin. The washed cells were collected, suspended in SDS-PAGE buffer, and then subjected to SDS-PAGE. The SUMO-3 molecule bound to the cells was detected by Western blotting with anti-FLAG M2 antibody.

\section{RESULTS AND DISCUSSION}

Secretion of SUMO Molecules from Cultured Cells Interferon-induced ubiquitin-like protein ISG15 is secreted from cultured cells upon interferon stimulation. ${ }^{112)}$ In the course of the transfection experiment, we noticed that SUMO was secreted from transfected cells. In this experiment,
FLAG-tagged SUMO-3 was transiently expressed in HeLa cells, and the culture medium was collected at various times and analyzed by Western blotting with anti-FLAG M2 antibody (Fig. 1A). One hour after the medium had been exchanged, the signal for SUMO-3 was detected in the culture medium and the level was increased in a time-dependent manner.

To determine whether SUMO-3 is secreted as a free form and/or a conjugated form, we constructed SUMO-3 mutants: $\Delta \mathrm{GG}$ mutant, which lacks the $\mathrm{C}$-terminal di-glycine and is incapable of being conjugated to target proteins, and K11R mutant, in which the lysine 11 residue had been changed to arginine and formation of the poly-SUMO chain is pre-

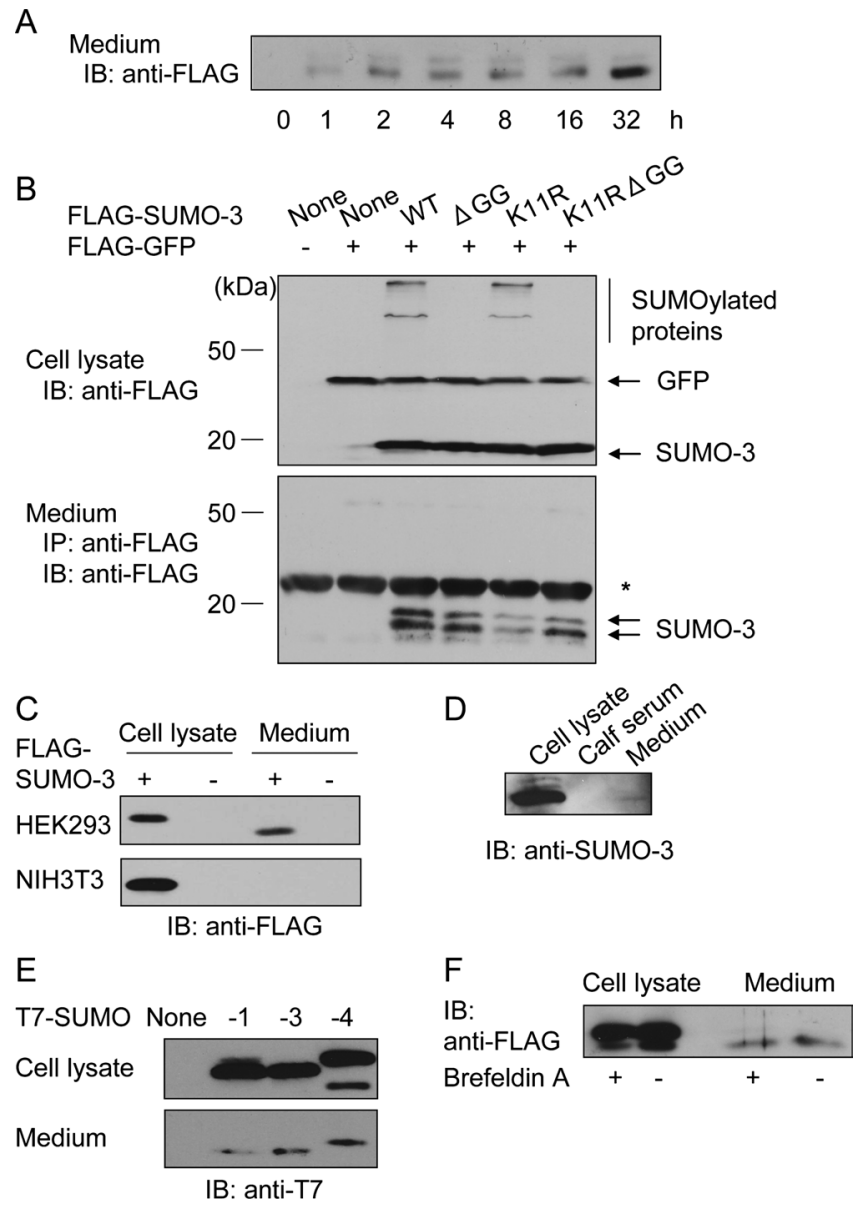

Fig. 1. SUMO-3 Is Secreted from HeLa Cells

(A) FLAG-tagged SUMO-3 was transiently expressed in HeLa cells. After the medium had been exchanged, the cells were cultured, and the culture media were collected at the indicated times, centrifuged to remove any cells, and subjected to SDSPAGE, followed by immunoblotting (IB) with anti-FLAG M2 antibody. (B) HeLa cells expressing FLAG-tagged SUMO-3 (WT) and its mutants ( $\triangle \mathrm{GG}, \mathrm{K} 11 \mathrm{R}$, and $\mathrm{K} 11 \mathrm{R} \Delta \mathrm{GG}$ ) and FLAG-tagged GFP were cultured for $36 \mathrm{~h}$. The culture media were collected, centrifuged to remove any cells, and subjected to immunoprecipitation (IP) with anti-FLAG M2 agarose beads, and the resulting precipitates were subjected to SDS-PAGE, followed by IB with anti-FLAG antibody (lower panel). On the other hand, the cells were lysed and the resulting lysate was subjected to SDS-PAGE, followed by IB with anti-FLAG antibody (upper panel). An asterisk indicates IgG light chain. Note that a doublet band was detected in the culture medium, where its upper band corresponds to the band detected in the cell lysate. (C) HEK293 and NIH3T3 cells expressing FLAG-tagged SUMO-3 were cultured for $36 \mathrm{~h}$. The culture medium and cell lysate were prepared as in (B) and subjected to IB with anti-FLAG antibody. (D) HeLa cells were cultured for $36 \mathrm{~h}$. The culture medium and cell lysate were prepared as in (B) and subjected to IB with anti-SUMO-3 antibody. (E) HeLa cells expressing T7-tagged SUMO- $1,-3$, and -4 were cultured for $36 \mathrm{~h}$. The culture medium and cell lysate were prepared as in (B) and subjected to IB with anti-T7 antibody. (F) HeLa cells expressing FLAG-tagged SUMO-3 were cultured in the presence of brefeldin A $(10 \mu \mathrm{g} / \mathrm{ml})$ for $36 \mathrm{~h}$. The culture medium and cell lysate were prepared as in (B) and subjected to IB with anti-FLAG antibody. 
vented. When HeLa cells were co-transfected with FLAGtagged wild-type SUMO-3 (WT) and FLAG-tagged GFP (control), a free form of SUMO-3 was detected in the culture medium (Fig. 1B, lower panel) as well as in the cell lysate (upper panel), while GFP, used as an intracellular marker, was detected in the cell lysate but not in the culture medium. In addition, the bands corresponding to proteins modified by SUMO-3 were detected in the cell lysate but not in the culture medium. In the cases of $\Delta \mathrm{GG}, \mathrm{K} 11 \mathrm{R}$, and $\mathrm{K} 11 \mathrm{R} \Delta \mathrm{GG}$ mutants, the results obtained in the culture media were found to be the same as that in the case of the wild type, confirming that SUMO-3 is secreted as a free form. It should be noted that the secreted SUMO-3 molecule was detected as a doublet band in either case (see lower panel in Fig. 1B) and that a ratio of the upper band to the lower band underwent changed irrespective of the same transfection procedures although the reason remains unclear. As shown in Fig. 1C, SUMO-3 was secreted from HEK293 cells as well as from HeLa cells but not from NIH3T3 cells. SUMO-3 was also secreted from H1299, A549, and MCF7 cells (data not shown).

To detect the secretion of endogenous SUMO-3 molecule, an antibody against SUMO-3 was prepared and the culture medium was subjected to SDS-PAGE and immunoblotting with the anti-SUMO-3 antibody (Fig. 1D). SUMO-3 was detected in the culture medium. Since the anti-SUMO-3 antibody used cross-reacts with SUMO-2 but not with SUMO-1 (data not shown), it is thought that the detected band is derived from SUMO-2 and -3. To confirm that other SUMO family members are also secreted, HeLa cells were transfected with T7-tagged SUMO-1, -3 , and -4 and the culture media were subjected to Western blotting with anti-T7 antibody (Fig. 1E). SUMO-1, -3 , and -4 were all detected in the respective culture media, suggesting that all SUMO family members are secreted.

Next, we investigated the effect of brefeldin A, an inhibitor of intracellular transport through the endoplasmic reticulum (ER)/Golgi pathway, on the secretion of SUMO-3 because SUMO lacks the signal sequence that is required for transport of nascent proteins through the ER membrane. As shown in Fig. 1F, secretion occurred despite the presence of brefeldin A, indicating that SUMO is secreted through a nonclassical pathway.

Thus, we have provided definitive evidence for the secretion of SUMO. We also investigated the secretion of ubiquitin and NEDD8, another type I ubiquitin-like protein, but we were unable to detect the secretion of either protein. The secretion of ISG15 is induced by interferon stimulation, but in the conditions we used, the secretion of SUMO was detected without any stimulus, suggesting that SUMO is secreted constitutively. Whether the secretion of SUMO is stimulated by a still-unknown stimulus is an interesting issue to be determined.

Enhancement of Cell Proliferation by Recombinant SUMO-3 Protein Since SUMO is secreted from cells as a free form but not as a conjugated or polymerized form, it is reasonable to assume that the secreted SUMO molecule has an extracellular role. In the case of ISG15, the recombinant protein can induce the secretion of interferon- $\gamma$ from $\mathrm{T}$ cells, leading to the stimulation of $\mathrm{NK}$ cells. ${ }^{12}$ ) To determine whether SUMO acts as a cytokine-like protein, recombinant SUMO-3 protein was added to NIH3T3 cells that had been

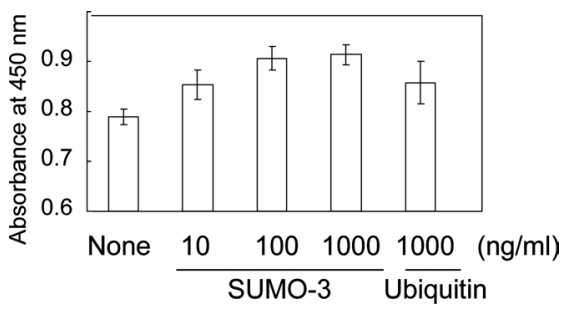

Fig. 2. SUMO-3 Has Proliferative Activity

NIH3T3 cells were treated with recombinant SUMO-3 and ubiquitin at the indicated concentrations for $48 \mathrm{~h}$. The absorbance at $450 \mathrm{~nm}$ was measured by the procedure described in Materials and Methods. Experiments were repeated four times and the error bars indicate standard deviation. Note that calf serum used in the cell culture was passed through an ultrafiltration filter to remove materials with molecular masses of less than $30 \mathrm{kDa}$.

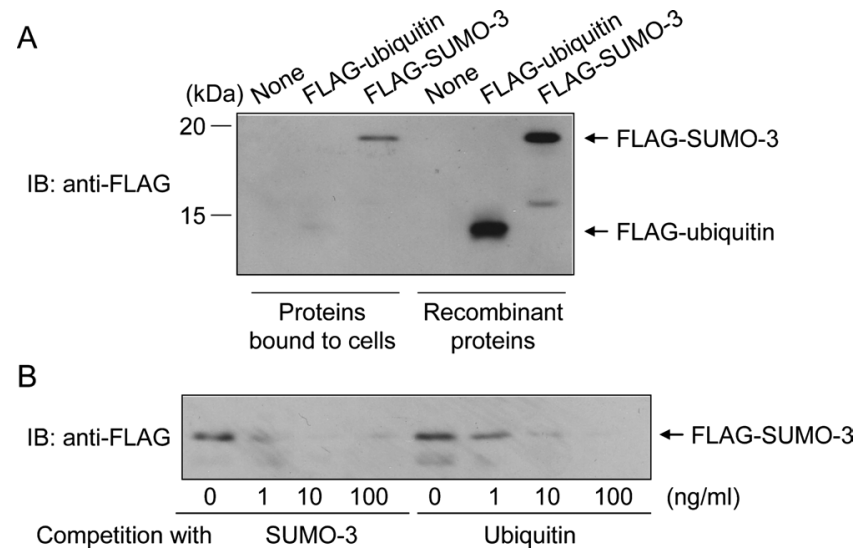

Fig. 3. SUMO-3 Binds to NIH3T3 Cells

(A) NIH3T3 cells were incubated with FLAG-tagged SUMO-3 $(1 \mathrm{ng} / \mathrm{ml})$ or FLAGtagged ubiquitin $(1 \mathrm{ng} / \mathrm{ml})$ for $10 \mathrm{~min}$. After washing, the cells were collected, suspended in SDS-PAGE buffer, and then subjected to SDS-PAGE. The SUMO-3 molecule bound to the cells was detected by IB with anti-FLAG antibody. (B) The SUMO-3 molecule bound to NIH3T3 cells in the presence of non-tagged SUMO-3 or ubiquitin at concentrations of 1,10 , and $100 \mathrm{ng} / \mathrm{ml}$ was detected as in (A).

found not to secrete SUMO-3 and the effect on the proliferation of the cells was analyzed (Fig. 2). It was found that recombinant SUMO-3 substantially enhances the proliferation of NIH3T3 cells. Ubiquitin also enhanced the cell proliferation, though to a lesser extent. It should be noted that the recombinant SUMO-3 protein was unable to enhance the proliferation of cells other than NIH3T3 cells (data not shown).

Our finding that SUMO-3 enhances proliferation of NIH3T3 cells implies the presence of a SUMO-3-binding site on the cell surface. To confirm this, we carried out a SUMO binding experiment: Recombinant FLAG-tagged SUMO-3 was added to NIH3T3 cells in the presence or absence of non-tagged recombinant SUMO-3. As shown in Fig. 3, binding of FLAGtagged SUMO-3 to the cells was detected under the experimental conditions used (A) and this binding was inhibited by the presence of non-tagged SUMO-3 (B). Interestingly, FLAG-tagged ubiquitin was also found to bind to the cells more weakly than FLAG-tagged SUMO-3 did (A) and the competitive ability of ubiquitin was weaker than that of SUMO-3 (B), possibly because of weak homology between SUMO-3 and ubiquitin. Taken together, the results suggest that a SUMO-3-binding site is present on the surface of NIH3T3 cells and that SUMO-3 acts as a cytokine-like protein through the putative binding receptor to enhance cell proliferation. 
C-terminal Sequence of SUMO-3 Governs Its Secretion and Cell Proliferative Activity Mouse SUMO-3 is originally translated as a precursor protein consisting of 110 amino acids and is then processed at the $\mathrm{C}$-terminal side of the di-glycine motif by SUMO-specific proteases, resulting in the production of a mature form of 92 amino acids. As shown in Fig. 1, the secreted form of SUMO-3 was detected as a doublet band, possibly consisting of the original mature form and/or processed forms. In addition, the $\Delta \mathrm{GG}$ mutant of SUMO-3 was found to be secreted to give a doublet band (see Fig. 1B). To determine whether the C-terminal sequence of SUMO-3 governs its secretion, we constructed C-terminally truncated mutants of SUMO-3, the N-termini of which had been tagged with FLAG tag sequences, and analyzed their secretion. The mutants used included ones containing and lacking the C-terminal tri-glutamine motifs, SUMO-3 $(1-89)$ and $(1-86)$, respectively, because the tri-glutamine motif plays an important role in maturation of SUMO, mediated by SUMO-specific proteases. ${ }^{14)}$ As shown in Fig. 4A, FLAG-tagged SUMO-3 mutants lacking the C-terminal sequences, SUMO-3 (1-79), (1-81), and (1-84), were unable to be secreted from the cells, whereas, on the other hand, FLAG-tagged SUMO-3 $(1-89)$ and $(1-86)$ were found to be secreted. It should be noted that the $\mathrm{C}$-terminal region is conserved between mouse and human SUMO-3 molecules. Thus, these results suggest that the C-terminal sequence of SUMO-3 governs its secretion. To obtain evidence for this assumption, we expressed a GFP-fusion protein in which GFP had been fused with the C-terminal sequence (85-92) of SUMO-3 and investigated the secretion, but we could not

A

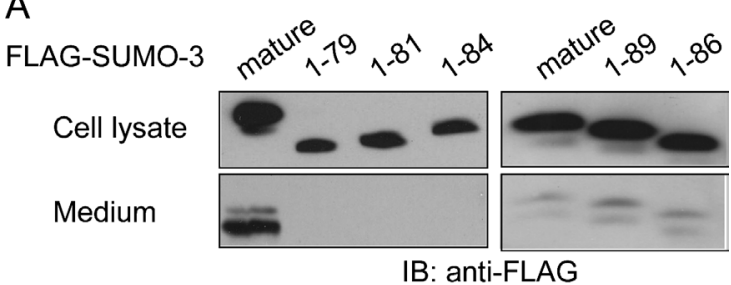

B

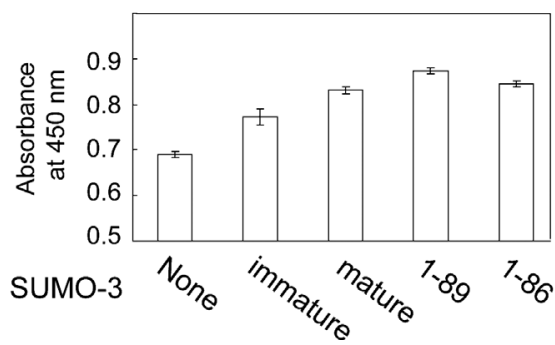

Fig. 4. The C-Terminal Sequence of SUMO-3 Has an Important Role in Its Secretion and Exhibition of Cell Proliferative Activity

(A) HeLa cells expressing FLAG-tagged mature SUMO-3 and its C-terminally truncated mutants, SUMO-3 $(1-79),(1-81),(1-84),(1-86)$, and $(1-89)$ were cultured for $36 \mathrm{~h}$. The culture medium and cell lysate were prepared as described in the legend to Fig. 1B and subjected to IB with anti-FLAG antibody. (B) NIH3T3 cells were treated with recombinant immature and mature SUMO-3 and its C-terminally truncated mutants, SUMO-3 (1-89) and (1-86), at concentrations of $1 \mu \mathrm{g} / \mathrm{ml}$ for $48 \mathrm{~h}$. The absorbance at $450 \mathrm{~nm}$ was measured by the procedure described in Materials and Methods. Experiments were repeated four times and the error bars indicate standard deviation. Note that calf serum used in the cell culture was passed through an ultrafiltration filter to remove materials with molecular masses of less than $30 \mathrm{kDa}$. obtain evidence for its secretion (data not shown). Further study is needed to elucidate the mechanism of secretion of SUMO, including the requirement of the C-terminal sequence.

Next, we analyzed the proliferative activity of C-terminally truncated mutants of SUMO-3, SUMO-3 (1-89) and (1-86), which had been demonstrated to be secreted. As shown in Fig. 4B, the truncated mutant SUMO-3 (1-89) showed stronger proliferative activity than that of the mature form. Thus, the secreted C-terminally truncated mutants of SUMO-3 act as cytokine-like proteins.

In the present study, we found the secretion of the SUMO molecule and its cytokine-like activity. To our knowledge, this is the first report on a novel extracellular role of SUMO family members. The secretion requires the C-terminal sequence of SUMO. In the secretion pathway of SUMO, this molecule is often cleaved in the C-terminal region and the secreted mature and processed forms show proliferative activity. It is possible that secreted SUMO binds to a putative SUMO-binding receptor in the cells to transduce a proliferative signal. In this study, we demonstrated that SUMO has cell proliferative and binding abilities toward NIH3T3 cells, suggesting that at least NIH3T3 cells have a SUMO-binding receptor. Identification of this putative SUMO-binding receptor will reveal the mechanism of SUMO-mediated cell proliferation.

Acknowledgements This study was supported in part by Grants-in-Aid for scientific research from the Ministry of Education, Culture, Sports, Science and Technology of Japan. The authors thank Dr. H. Ariga of Hokkaido University for the SUMO-1 mammalian expression plasmid.

\section{REFERENCES}

1) Johnson E. S., Annu. Rev. Biochem., 73, 355-382 (2004).

2) Hay R. T., Mol. Cell, 18, 1-12 (2005).

3) Kerscher O., Felberbaum R., Hochstrasser M., Annu. Rev. Cell Dev Biol., 22, 159-180 (2006).

4) Okuma T., Honda R., Ichikawa G., Tsumagari N., Yasuda H., Biochem. Biophys. Res. Commun., 254, 693-698 (1999).

5) Uchimura Y., Nakao M., Saitoh H., FEBS Lett., 564, 85-90 (2004).

6) Baba D., Maita N., Jee J.-G., Uchimura Y., Saitoh H., Sugasawa K., Hanaoka F., Tochio H., Hiroaki H., Shirakawa M., Nature (London), 435, 979-982 (2005).

7) Baba D., Maita N., Jee J.-G., Uchimura Y., Saitoh H., Sugasawa K., Hanaoka F., Tochio H., Hiroaki H., Shirakawa M., J. Mol. Biol., 359, 137-147 (2006).

8) Desterro J. M., Rodriguez M. S., Hay R. T., Mol. Cell, 2, 233-239 (1998).

9) Hoege C., Pfander B., Moldovan G. L., Pyrowolakis G., Jentsch S., Nature (London), 419, 135-141 (2002).

10) Steffan J. S., Agrawal N., Pallos J., Rockabrand E., Trotman L. C., Slepko N., Illes K., Lukacsovich T., Zhu Y. Z., Cattaneo E., Pandolfi P. P., Thompson L. M., Marsh J. L., Science, 304, 100-104 (2004)

11) D’Cunha J., Ramanujam S., Wagner R. J., Witt P. L., Knight E., Jr., Borden E. C., J. Immunol., 157, 4100-4108 (1996).

12) D'Cunha J., Knight E., Jr., Haas A. L., Truitt R. L., Borden E. C., Proc. Natl. Acad. Sci. U.S.A., 93, 211-215 (1996).

13) Nakagawa K., Yokosawa H., FEBS Lett., 530, 204-208 (2002).

14) Owerbach D., McKay E. M., Yeh E. T., Gabbay K. H., Bohren K. M., Biochem. Biophys. Res. Commun., 337, 517-520 (2005). 